\title{
Las estrategias fiscales como respuesta a un contexto local en transformación en España
}

Clemente J. Navarro Yáńez*

M. Ángeles Huete García**

Palabras clave: Crisis del Estado de bienestar, nueva gobernanza local, estrategias fiscales, dinámica local, dinámica intergubernamental, tamaño municipal.

\section{Introducción. La Administración local ante un contexto en cambio $^{1}$}

Es sabido que la pasada década de los setenta supuso el inicio de cambios significativos en el ámbito económico, social y político que dieron lugar al planteamiento de síntomas de crisis en el Estado de bienestar, y con ello, a iniciativas que, respondiendo a nuevas formas en la concepción de sus funciones y responsabilidades, así como la gestión de la Administración pública, han ido progresivamente transformándolo².

Ahora bien, dichas transformaciones se manifiestan de manera especial en el ámbito local y tienen también en este contexto respuestas concretas que, bajo el calificativo de nuevo localismo o nueva gobernanza local, vienen desarrollándose desde hace décadas. En concreto, estas respuestas se dan tanto en su dimensión política como en su dimensión administrativa (Navarro 2006: 7). Por una parte, el gobierno local se presenta como un actor relacional que gobierna a través de redes de actores (Blanco y Gomá 2002: 23), estableciendo procesos de colaboración tanto con la sociedad civil, a través de sus actores socioeconómicos, como con otros niveles de gobierno. Por otra parte, en el ámbito local se han venido implementando nuevas formas de gestión pública para incrementar la efi-

* Profesor Titular de Sociología de la Universidad Pablo de Olavide de Sevilla. Director del Centro de Sociología Politica y responsable del Observatorio del Mundo Local. Director del proyecto Fiscal Austerity and Urban Innovation en España.

** Profesora Ayudante de Sociología de la Universidad Pablo de Olavide de Sevilla. Investigadora del Centro de Sociología Politica, adscrita al Observatorio del Mundo Local. Miembro del proyecto Fiscal Austerity and Urban Innovation en España.

${ }^{1}$ Los autores desean agradecer a los evaluadores y especialistas que con sus correcciones y sugerencias han contribuido a mejorar sustancialmente este artículo.

2 Sobre la crisis del Estado de bienestar e iniciativas de reforma puede consultarse, entre otros, Mishra (1992) y Offe (1990). 
cacia y eficiencia en la prestación de los servicios públicos, dadas a conocer posteriormente con el nombre genérico de «Nueva Gestión Pública» en su aplicación a otros niveles de gobierno y administraciones. En su conjunto, la llamada nueva gobernanza local constituye un movimiento estratégico de adaptación a un contexto en cambio; tanto a través de nuevas formas de gobernar el ámbito local a través de fórmulas de colaboración con otros actores, como de gestionar su administración mediante nuevas técnicas de gestión pública. Lo que suele reflejarse en términos de estrategias fiscales, esto es, orientaciones y acciones adoptadas por la hacienda pública local que se orientan hacia ello.

Ahora bien, la nueva gobernanza local, y la forma concreta que adopta en términos de estrategias fiscales, en buena medida se explica por la capacidad de respuesta que cada ente local tenga a su alcance, por su estructura de oportunidades de acción; la cual vienen dada, fundamentalmente, por la dinámica socio-económica local, el diseño intergubernamental en el que se sitúan los municipios. Sin menoscabo de que en ello también cuentan las visiones y preferencias de las autoridades políticas y administrativas municipales que actúan en tal contexto.

¿Cómo se manifiesta la nueva gobernanza local en términos de estrategias fiscales entre los municipios españoles?; esto es, ¿qué estrategias fiscales vienen utilizando? Pero además, ¿̨a qué responde el uso de unas u otras estrategias? Tratar de aportar algunas evidencias en torno a estas preguntas es el objeto del presente trabajo. Para ello se estructura en cuatro apartados. El primero revisa los principales modelos explicativos utilizados en otros estudios, par tratar de extraer algunas ideas analíticas aplicables al caso español. El segundo presenta la estrategia de indagación y el corpus empírico utilizado. El tercero presenta evidencias empíricas para el caso español, primero sobre la extensión en el uso de diversas estrategias fiscales entre municipios españoles de 10.000 o más habitantes, y después sobre algunos factores explicativos. Pasando en el cuarto y último apartado a presentar algunas conclusiones al respecto.

\section{Nueva gobernanza local como estrategias fiscales}

Tal y como se ha señalado en el apartado anterior, en el marco de la nueva gobernanza local se producen una serie de respuestas a cambios en el contexto que son interpretados por los responsables locales como presiones o retos (Eulau y Eyestone 1968). Dichas reformas se implementan con el objetivo de lograr una adaptación a los cambios a corto plazo y para asegurar supervivencia a largo plazo (Glueck 1972; Hambrick 1981; McGowan y Stevens 1983).

Buena parte de las presiones a las que se enfrentan los gobiernos locales se producen como resultado de un proceso de declive económico que les somete a presiones fiscales, ante lo que desarrollan una variedad de estrategias fiscales (Clark y Ferguson 1983; Mouritzen 1992), entendidas como cursos de acción adoptados por los gobiernos locales para aliviar las presiones financieras percibidas e interpretadas por los representantes políticos como elementos que tienen un efecto adverso en la estabilidad e integridad financiera de las ciudades a largo plazo (Pammer 1990: 16). Estas estrategias fiscales pueden orientarse bien a la reducción del gasto, a través de recortes en la prestación de servicios, en gastos administrativos o de personal, por ejemplo; o bien al aumento de ingresos, ya sean propios a través del incremento de impuestos o tasas, ya sea a través de fuentes de financiación procedentes de otras Administraciones. ¿Qué suele explicar la puesta en marcha de tales estrategias?

Son numerosas las aportaciones destinadas a comprender las causas por las que las Administraciones locales ponen en marcha diferentes estrategias fiscales en contextos de transformación que suponen las sociedades contemporáneas. Para tratar de obtener una visión global de ellos quizás puedan agruparse en atención ala importancia que 
conceden a un factor explicativo en particular, ya sea situado en el ámbito municipal -o nivel micro-, ya sea en relación al diseño intergubernamental en el que se sitúan los municipios -o nivel marco- ${ }^{3}$.

Uno de los factores a los que se ha concedido capacidad explicativa es el relacionado con la visión de las propias autoridades políticas y administrativas municipales, ya sea en términos de 'cultura política', ya sea en términos de 'percepción de crisis fiscal'. Entre los primeros cabe situar las investigaciones desarrolladas por Clark (1994), para quien las estrategias fiscales se relaciona fundamentalmente con ideologías, partidos y culturas políticas. Así, los municipios desarrollan diferentes estrategias fiscales en función de las preferencias de sus líderes políticos, ya sean éstos de 'izquierda', entre los que prevalece una tendencia a un mayor gasto de corte redistributivo, ya sean de 'derecha', para los que se trata, sobre todo, de establecer recortes en los servicios y la privatización para reducir el gasto público, o bien se acerquen al modelo de la 'nueva cultura política', cuyos líderes se caracterizan por tratar de reducir el gasto pero sin recortar servicios principalmente mediante estrategias que apuntan a un incremento de la productividad mediante nuevas técnicas de gestión o mediante la colaboración público-privada ${ }^{4}$.

Pero también se ha podido constatar que la percepción de crisis fiscal por parte de actores claves del municipio (el alcalde o funcionarios directivos), con cierta independencia de que esta exista o no realmente, influye en la puesta en práctica de determinadas estrategias fiscales. Así, la percepción sobre elementos tales como las restricciones en las transferencias procedentes de niveles superiores de gobierno, la inflación, el desempleo y las demandas de los empleados municipales o de la ciudadanía, parecen influir en la adopción de unas u otras estrategias fiscales (Pammer 1990).

Sin duda, los cambios reales en tales aspectos son también factores significativos a la hora de explicar el impulso de diferentes estrategias, ya sea bien en dinámica socio-económica y socio-política local, bien en el marco intergubernamental. Así, diversos estudios, como los realizados por Nivola (1982) o Stein (1984), han considerado que el tamaño de la población, la riqueza del municipio, las demandas sociales existentes (ocasionadas por la inmigración o la presencia de otro tipo de población dependiente) o la situación geográfica del municipio, son aspectos explican la puesta en marcha de determinados mecanismos para afrontar la adaptación a contextos locales en cambio. Y en concreto, el uso de estrategias de austeridad fiscal se relaciona, sobre todo, con un marco socio-económico municipal en declive.

No obstante, el marco intergubernamental también parece incidir en ello, tanto en la forma en que se organiza la división del trabajo entre esfera política y administrativa a nivel municipal, a lo que suele denominarse 'forma de gobierno' local (Mouritzen y Svara 2002), como en la relación entre municipios y otras Administraciones. Respecto a la primera cuestión, cuando la forma de gobierno es la del denominado 'strong mayor', según el cual el alcalde es el líder político y administrativo, suele darse un mayor gasto, mientras que entre los municipios que adoptan al forma de 'council-manager', en cuyo caso un gestor es el responsable de la Administración y el alcalde del gobierno municipal, suelen asociarse con un mayor uso de estrategias de austeridad fiscal (Pammer 1990; Miranda 1994). Pero parece ser la pérdida de ingresos de otras Administraciones, y sobre todo, la dependencia de éstas de la estructura de ingresos y de la discrecionalidad impositiva, el factor que en mayor medida afecta a la situación fiscal de los municipios (Mouritzen 1992), y con ello, a la implementación de nuevas estrategias fiscales (Stein 1984). De esta forma, el marco intergubernamental pasa a ser un factor clave en la estructura de oportunidades de acción municipal común a los municipios de un mismo país.

${ }^{3}$ En lo que sigue debe considerarse que la mayor parte de los estudios se han realizado en Estado Unidos, que presenta unos rasgos en su sistema de gobierno local diferentes del español. Aún así, pueden extraerse algunos argumentos analíticos de utilidad para su análisis.

4 Para una visión general de la relación entre gasto público y el esquema izquierda-derecha véase Jens Blom-Hansen, et al. (2006). 
En este sentido, y antes de pasar al análisis empírico del caso español, cabe preguntarse: ¿Qué rasgos institucionales presenta el sistema de gobierno local en España como elemento común a la estructura de oportunidades de acción de los municipios?

\section{Estrategias fiscales en los municipios españoles: diseño del estudio}

De lo apuntado en el apartado anterior cabe indicar que el marco intergubernamental puede tener un peso explicativo relevante en la extensión de determinadas estrategias fiscales, con cierta independencia de otros factores situados en el ámbito local (actores relevantes o dinámica socio-económica). Es por ello que en este aparatado se presentan, en primer lugar, el marco en el que se desarrolla la indagación, esto es, se presentan, brevemente, algunos rasgos del sistema de gobierno local en relación con el análisis de las estrategias fiscales que puedan desarrollar. En segundo lugar se presenta el diseño realizado para analizar la extensión en el uso de diversas estrategias, así como factores explicativos que puedan dar cuenta de ello.

\subsection{Estructura de oportunidades institucionales para los municipios españoles: la 'lógica de la dependencia'}

El sistema de gobierno local español, junto con otros países del sur de Europa, se caracteriza por presentar un modelo de «jerarquías fundidas» (Navarro y Ramírez 2003), en donde, reconocida la capacidad del municipio para prestar servicios a la ciudadanía, distintas Administraciones interactúan en el en el diseño y ejecución de políticas en su territorio. Ello hace que en el ámbito local se desarrollen intensos procesos de interacción entre las Administraciones locales y otros niveles de gobierno.

Sin embargo, a pesar de tener reconocido un importante papel desde el punto de vista político, o 'localismo político', que dirían Page y Goldsmith (1987), tienen un escaso protagonismo administrativo, o 'localismo legal'. De hecho, las entidades locales españolas sólo gestionan alrededor del 13\% del gasto público total, lo que supone una limitada capacidad para llevar a cabo la gestión de aquellas responsabilidades que formalmente tiene reconocidas. De esta forma, la posición que los actores locales ocupan dentro del entramado institucional español (con capacidades políticas formalmente reconocidas, pero con limitada capacidad de gestión a tenor de sus limitados recursos) configura un sistema de relaciones intergubernamentales basado en la dependencia financiera entre el gobierno local y otros niveles de gobierno. Y ello tanto desde el punto de vista de los ingresos propios, cuya naturaleza y volumen se determina en niveles superiores de gobierno, como desde el punto de vista de las transferencias procedentes de otros niveles de gobierno. Situación que no ha variado a lo largo de los años, a pesar de la reforma de la financiación local acometida en el año 2002 (Ley 51/2002) por la que se establecía como objetivo fundamental mantener y fortalecer la garantía del principio de suficiencia financiera de las entidades locales e incrementar la autonomía municipal en el ámbito de los tributos locales (de León y Dorta 2005).

Tal como muestra la Tabla 1, los ingresos locales se estructuran en relación a dos dimensiones fundamentales; por un lado los ingresos propios (impuestos, tasas y otros tributos), que representan prácticamente la mitad de los ingresos locales, y las transferencias procedentes de otros niveles de gobierno, que suponen más de un tercio de los ingresos. (Ahora bien, aunque los impuestos siguen suponiendo una parte importante de las finanzas locales, se siguen estableciendo limitaciones en su naturaleza, lo cual de nuevo pone de manifiesto la limitada autonomía del 
gobierno local para decidir sobre sus ingresos ${ }^{5}$ ); y por otro la estructura de ingresos locales, que refleja una significativa dependencia financiera hacia otros niveles de gobierno desde los cuales se siguen financiando buena parte de los servicios públicos locales gracias a las transferencias de naturaleza finalista.

\section{TABLA 1}

\section{Estructura de ingresos de los municipios españoles. Año 2004}

\begin{tabular}{|l|r|}
\hline Capítulos & Municipios \\
\hline Impuestos directos & 10.600 .591 \\
\hline Impuestos indirectos & 2.207 .207 \\
\hline Tasas y otros ingresos & 7.027 .992 \\
\hline Transferencia corrientes & 10.840 .938 \\
\hline Ingresos patrimoniales & 1.050 .474 \\
\hline Enajenación de inversiones reales & 2.741 .370 \\
\hline Transferencias de capital & 3.494 .628 \\
\hline Activos financieros & 97.843 \\
\hline Pasivos financieros & 3.452 .615 \\
\hline Total Ingresos & 41.513 .657 \\
\hline
\end{tabular}

Fuente: Ministerio de Economía y Hacienda. Estadísticas territoriales.

No obstante, al igual que sucede en otros países, la agenda de actuación de los gobiernos municipales españoles se centra en la provisión de servicios urbanos de producción, o dicho de otra forma, destinados al funcionamiento del municipio (agua, luz, limpieza viaria), y cada vez en mayor medida, en la provisión de servicios de bienestar (Navarro 1998). Dichos asuntos se sitúan dentro de su ámbito de responsabilidad a tenor de las competencias atribuidas al nivel local que se han ido incorporando como consecuencia de la denominada «segunda descentralización» (Suárez 2001), así como de las nuevas demandas que, como consecuencia de importantes cambios en la sociedad, se le plantean a la Administración local. Dichas demandas suponen nuevos retos a la capacidad de respuesta de las Administraciones locales y se circunscriben a asuntos tan variados como la inmigración, la participación ciudadana o el medio ambiente. De esta forma, los municipios españoles prestan una serie de servicios, a menudo denominados «impropios», (Consejería de Gobernación 2002) que estrictamente no les corresponden pues no cuentan con la competencia exclusiva ni expresa y para los que, por tanto, no cuentan con financiación alguna.

Así, el sistema de gobierno local español sitúa los municipios ante el denominado «dilema de la autonomía local»; a saber, el reconocimiento o consenso de que en razón de su cercanía debe ser la unidad de gobierno que preste los servicios a la ciudadanía, frente a su escasa capacidad financiera y/o su dependencia de otras unidades de gobierno a este respecto (Ylönen 1989), si se quiere, un marco en el que cabe esperar la extensión de nuevas estrategias fiscales, pero también una relativa importancia de aquellas relacionadas con la obtención de ingresos procedentes de otras administraciones.

${ }_{5}^{5}$ Un ejemplo de ello, supone la decisión de eliminar el Impuesto de Actividades Económicas que hasta la reforma del año 2002 suponía una parte importante de los ingresos locales a partir de la actividad económica del municipio. 


\subsection{Estrategia de investigación: encuesta de «Estrategias Fiscales en la Administración Municipal»}

La cuestión es, dado tal contexto, ¿qué estrategias fiscales han desarrollado los municipios? Para conocer este asunto bien es cierto que el análisis secundario de datos sobre las haciendas locales pudiera dar cuenta de orientaciones básicas de las estrategias fiscales, por ejemplo, mediante el análisis de la evolución de sus estructuras de ingresos y gastos. Ahora bien, ello no ofrece la posibilidad de analizar las estrategias fiscales, sino sus resultados sobre las haciendas locales; esto es, no permite conocer qué estrategias o cursos de orientación específicos han puesto en marcha de forma intencional los municipios para modificar su estructura de ingresos o gastos 6 .

En cambio, mediante encuestas sobre la importancia en el uso de diversas estrategias fiscales entre actores claves en el proceso presupuestario municipal puede ofrecerse información válida sobre la orientación o intencionalidad de la estrategia fiscal que, en general, ha puesto en marcha el municipio. Es por ello que esta ha sido la estrategia utilizada en el presente estudio mediante la aplicación de la 'Encuesta de Estrategias Fiscales en la Administración Municipal' del proyecto internacional Fiscal Austerity and Urban Innovation entre interventores/as de municipios españoles de 10.000 y más habitantes?. La encuesta, mediante envío postal, se realizó entre finales de 2003 e inicios de 2004, habiendo obtenido una tasa de respuestas del 45\% (300 municipios sobre el total de 664), lo que supone un error muestral del $5 \%$ para la muestra en su conjunto, cuya distribución por tamaño de hábitat no presenta sesgos apreciables (Tabla 2).

\section{TABLA 2}

Marco muestral y muestra por tamaño de bábitat

\begin{tabular}{|l|c|c|c|}
\hline \multirow{2}{*}{ Estudio } & \multirow{3}{*}{ Tamaño hábitat } & \multicolumn{2}{|c|}{$\begin{array}{c}\text { España > 10 mil } \\
(\mathbf{N}=\mathbf{6 4 4})\end{array}$} \\
\cline { 3 - 4 } & & $\mathbf{N}(\%)$ & $\mathbf{n}(\%)$ \\
\hline \multirow{2}{*}{ Estrategias fiscales } & $10-50 \mathrm{mil}$ & $542(81,6 \%)$ & $244(81,3 \%)$ \\
\cline { 2 - 4 } & $>50 \mathrm{mil}$ & $112(18,4 \%)$ & $56(18,7 \%)$ \\
\hline \multirow{2}{*}{ Total } & $664(100,0 \%)$ & $300(100,0 \%)$ \\
\hline
\end{tabular}

Fuente: Encuesta sobre estrategias fiscales en la Administración Municipal. (CSP-UPO).

El cuestionario preguntaba acerca del uso de diversas estrategias fiscales concretas relativas a orientaciones más genéricas en la gestión fiscal de los municipios, como son el aumento de los ingresos y la reducción del gasto. En concreto, se les propia la siguiente pregunta a los entrevistados: 'Los municipios suelen usar algunas de las estrategias fiscales que se presentan en la lista siguiente. ¿¿e ha hecho uso de ellas en este municipio?, y en su caso, en términos monetarios, een qué medida han sido importantes cada una de ellas en su municipio desde 1999?'. Pudiendo utilizar una de las siguientes categorías de respuesta en forma de escala ordinal: 'una de las más importantes', 'muy importante', 'algo importante', 'una de las menos importantes' y 'no se ha usado'. Y naturalmente, a grandes rasgos, las estrategias se referían a la intención de aumentar ingresos o bien a la de reducción de gastos, tal y como se muestra en la Tabla 3.

${ }^{6}$ Podríamos conocer si han aumentado -o no- las transferencias intergubernamentales, pero no si esa era un objetivo del municipio, o si ello se ha hecho a través de acuerdos para la prestación de servicios. Del mismo modo, podría saberse que ha habido una reducción de gastos, pero no la estrategia intencional utilizada para ello (recortes en personal, servicios, gastos administrativos, acuerdos con actores socio-económicos o nuevas estrategias de gestión).

7 Esta encuesta forma para del proyecto 'Bienestar y Sociedad Política Local' (SEC-2002-01936) realizado desde en el marco del Observatorio del Mundo Local por el Centro de Sociología Política de la Universidad Pablo de Olavide (CSP-UPO, en adelante). 


\section{TABLA 3 \\ Estrategias fiscales incluidas en el cuestionario}

\begin{tabular}{|c|c|}
\hline Estrategias de aumento de ingresos & Estrategias de reducción de gasto \\
\hline Buscar nuevas fuentes de ingresos para el municipio & Transferir responsabilidades o servicios a otras administraciones (regional o central) \\
\hline Aumentar transferencias intergubernamentales & Acuerdos con otros ayuntamientos para la prestación de servicios \\
\hline Incrementar impuestos municipales & Acuerdos con administración central para la prestación de servicios \\
\hline Obtener fondos de la Unión Europea & Acuerdos con admón autonómica para prestación servicios \\
\hline Incrementar cuotas o tasas por los servicios municipales & Prestación servicios mediante acuerdos con empresas privadas \\
\hline Vender suelo público municipal & Prestación servicios mediante acuerdos con asociaciones (ONGs, tercer sector...) \\
\hline Vender otros activos y bienes & Recortes presupuestarios en todas las áreas \\
\hline Posponer algunos pagos al siguiente año fiscal & Recortes presupuestarios en áreas o departamentos menos eficientes \\
\hline \multirow[t]{19}{*}{ Incrementar deuda a corto plazo } & Reducción de personal \\
\hline & Reducir gastos administrativos (no de los servicios) \\
\hline & Reducir niveles de prestaciones laborales \\
\hline & Congelar sueldos y salarios de empleados \\
\hline & No incrementar el número de trabajadores municipales \\
\hline & Reducir personal con jubilaciones \\
\hline & Reducir los gastos para equipamiento y viajes \\
\hline & Reducir servicios financiados a través de ingresos propios \\
\hline & Reducir servicios financiados a través de ingresos intergubernamentales \\
\hline & Rediseño procesos de trabajo para aumentar productividad \\
\hline & Mejoras en gestión administrativa para aumentar productividad \\
\hline & Eliminar programas (proyectos, servicios...) \\
\hline & Reducir los gastos de inversión \\
\hline & Mantener los incrementos en el gasto por debajo de la tasa de inflación \\
\hline & Promover jubilaciones anticipadas \\
\hline & Reducir horas extra de trabajo \\
\hline & Establecer acuerdos intermunicipales para compra de equipamientos, materiales... \\
\hline & Diferir el mantenimiento de los activos del municipio \\
\hline & $\begin{array}{l}\text { Imponer controles sobre nuevas construcciones para limitar directamente el crecimiento de } \\
\text { la población }\end{array}$ \\
\hline
\end{tabular}

Fuente: Elaboración propia.

Para conocer la extensión en el uso de cada estrategia, y con el objeto de facilitar su lectura e interpretación, cada respuesta se ha estandariza en escala $(0,100)$, donde el primer valor se corresponde a la categoría de respuesta 'no se ha usado' y el valor 100 a la de 'una de las más importantes'. Con el objeto de conocer la existencia de estra- 
tegias concretas se han realizado análisis factoriales de componentes principales, pudiendo validar así la elaboración de índices sintéticos para cada una de las dimensiones resultantes ${ }^{8}$.

Esto permitirá conocer la presencia y extensión en el uso de diversas estrategias fiscales para el conjunto de los municipios españoles de 10.000 o más habitantes. Para acercarnos a posibles razones sobre la variablidad en el uso entre los municipios, y en consideración a lo planteado en el segundo apartado, se han tenido en cuenta las siguientes variables: tamaño del municipio, preferencias de política pública del partido que gobierna el municipio y percepción sobre problemas que puedan afectar a la hacienda municipal. Para medir el segundo aspecto se han tomado la puntuación del partido del alcalde en la escala 'izquierda-derecha' elaborada en el marco del Party Manifesto Project (Budge, et al. 2001; Castle y Mair 1984). Para el caso de los problemas fiscales se incluyó una pregunta al respecto en el cuestionario: 'En los últimos tres años, ¿en qué medida han sido importantes los siguientes asuntos para las finanzas del municipio?', pudiendo indicar los entrevistados: 'uno de los menos importantes', 'algo importante', 'muy importante' o 'uno de los más importantes', estandarizando las respuestas en escala $(0,100)$ al igual que en las relativas a las estrategias fiscales. Los ítems incluidos son los que se muestran en la Tabla 4, agrupados en los cuatro aspectos teóricamente considerados: pérdida de ingresos, aumento de servicios, aumento de servicios, dinámica socio-económica y dinámica socio-política. Una vez elaborados estos indicadores sintéticos para cada uno de estos aspectos, junto al tamaño y el partido del gobierno municipal, se han realizado regresiones múltiples para conocer su capacidad explicativa sobre cada una de las estrategias fiscales identificadas anteriormente.

\section{Estrategias fiscales entre los municipios españoles}

En este apartado se presentan los resultados de los análisis realizados. En primer lugar se atenderá a los problemas fiscales que son identificados y su importancia, en segundo lugar, a las estrategias fiscales existentes y su extensión, y por último, a la medida que posprimeros explican la extensión de las segundas, junto con el tamaño del municipio y el partido que encabeza el gobierno municipal.

\subsection{Los problemas fiscales: sobre el 'dilema de la autonomía local'}

La Tabla 4 muestra la agrupación de los problemas en los cuatro tipos de problemas considerados. Puede apreciarse que, desde al perspectiva de los entrevistados, los municipios españoles presenta el anteriormente referido 'dilema de la autonomía local', un aumento en los servicios a prestar frente a una pérdida (o cuando menos, no aumento) de los ingresos municipales.

De hecho, en gran medida debido a su cercanía a la ciudadanía, las Administraciones municipales en España han visto aumentar las demandas que les dirigen los ciudadanos en los veinticinco años de gobiernos municipales democráticos incrementar, referidas tanto a servicios básicos y tradicionales, como a otros nuevos relacionados con el bienestar (servicios sociales, inmigración, igualdad) o socio-cultural (servicios deportivos, culturales, de ocio y recreativos,..). Además, a lo largo de los años últimos años se observa que se ha llevado a cabo un proceso descentralización desde las Administraciones central y autonómica, la denominada «segunda descentralización», que consiste en la transferencia paulatina de responsabilidades en relación con la prestación de servicios. Pero, por lo general, esta nueva distribución de responsabilidades entre los diferentes niveles de gobierno no tiene plasmación

${ }^{8}$ Los resultados de los análisis factoriales y la validación de los indicadores mediante correlación inter-items se muestran en el anexo. En concreto, Tabla A para problemas fiscales, Tabla B para estrategias de ingreso y Tabla C para estrategias de gasto. 


\section{TABLA 4}

Problemas fiscales: factores que influyen en las finanzas locales

\begin{tabular}{|c|c|c|c|}
\hline Indicadores & Variables incluidas & $\begin{array}{c}\text { Media } \\
(0,100)\end{array}$ & $\begin{array}{c}\text { Media } \\
(0,100)\end{array}$ \\
\hline \multirow{4}{*}{ Pérdida de ingresos } & Pérdida de ingresos del gobierno central & 48,54 & \multirow{4}{*}{46,75} \\
\hline & Pérdida de ingresos del gobierno autonómico & 42,23 & \\
\hline & Disminución de ingresos propios & 50,34 & \\
\hline & Limitación de ingresos o gastos por el gobierno central o autonómico & 45,89 & \\
\hline \multirow{2}{*}{ Aumento de los servicios } & Aumento demanda servicios por los ciudadanos & 73,88 & \multirow{2}{*}{62,11} \\
\hline & Costes por la transferencia de servicios del estado o la autonomía al municipio & 50,34 & \\
\hline \multirow{2}{*}{ Dinámica socio-económica } & Inflación & 32,73 & \multirow{2}{*}{32,23} \\
\hline & Desempleo & 31,73 & \\
\hline \multirow{3}{*}{ Dinámica sociopolítica } & Imposibilidad de acuerdos con otros municipios para servicios conjuntos & 26,81 & \multirow{3}{*}{29,88} \\
\hline & Presiones de los contribuyentes para reducir impuestos & 29,65 & \\
\hline & Presiones de empleados municipales & 33,18 & \\
\hline
\end{tabular}

Fuente: Encuesta sobre Estrategias Fiscales en la Administración Municipal (CSP-UPO).

práctica, a lo que debe añadirse la existencia de regulaciones que limitan la capacidad de ingreso de los municipios (seguramente en atención a la supresión de impuestos como el IEA). Aspectos éstos que pueden incidir en una percepción de crisis fiscal en la forma del dilema de la autonomía local, tal y como muestran los resultados presentados más arriba.

Unos resultados que, en cualquier caso, son los esperados a tenor del marco institucional en el que se sitúan los municipios españoles, si bien tampoco parece despreciable la importancia de otros factores relativos a la dinámica socioeconómica local, que remiten a la imposibilidad de recabar ingresos propios, o bien al clima sociopolítico, ya sea por la relación con otros municipios o la ciudadanía, ya sea por rigideces derivadas de presiones o conflictos sociolaborales internos.

\subsection{La naturaleza y extensión de las estrategias fiscales}

Si esta es la naturaleza de la percepción del contexto fiscal de los municipios, ¿qué estrategias fiscales desarrollan? En cuanto a las estrategias de ingreso, los análisis muestran la existencias de cuatro grandes tipos: incremento de transferencias, de ingresos propios, uso o incremento de deuda pública y venta de recursos y bienes públicas. Su extensión media entre los municipios se muestra en la Tabla 5.

Puede apreciarse que destacan, en primer lugar, estrategias fiscales destinadas al incremento de los ingresos propios, seguidas a aquellas destinadas a incrementar las transferencias, fundamentalmente procedentes de otros niveles de gobierno. Son las estrategias menos utilizadas las de incremento de la deuda y, finalmente, aquéllas en las que se obtienen recursos a partir de la venta de recursos públicos, fundamentalmente referidos a la venta de suelo. En virtud de los resultados obtenidos, se muestra una tendencia de continuación en el tipo de estrategias de ingre- 


\section{TABLA 5}

Estrategias de aumento de ingresos

\begin{tabular}{|c|c|c|c|}
\hline Indicadores & Variables incluidas en el indicador & $\begin{array}{c}\text { Media } \\
(0,100)\end{array}$ & $\begin{array}{c}\text { Media } \\
(0,100)\end{array}$ \\
\hline \multirow{2}{*}{ Incremento de ingresos propios } & Incrementar impuestos & 48,76 & \multirow{2}{*}{48,51} \\
\hline & Incrementar cuotas o tasas por servicios & 48,26 & \\
\hline \multirow{3}{*}{ Incremento de la deuda } & Incrementar deuda a corto plazo & 25,70 & \multirow{3}{*}{35,15} \\
\hline & Posponer pagos al siguiente año fiscal & 30,93 & \\
\hline & Incrementar deuda a largo plazo & 48,84 & \\
\hline \multirow{3}{*}{ Incremento de las transferencias } & Aumentar transferencias intergubernamentales & 43,51 & \multirow{3}{*}{44,53} \\
\hline & Buscar nuevas fuentes de ingresos & 54,27 & \\
\hline & Obtener fondos de la Unión Europea & 35,82 & \\
\hline \multirow{2}{*}{ Venta de recursos y bienes públicos } & Vender suelo público & 41,95 & \multirow{2}{*}{27,56} \\
\hline & Vender otros activos y bienes & 13,17 & \\
\hline \multicolumn{3}{|l|}{ Media total estrategias de ingreso } & 38,93 \\
\hline
\end{tabular}

Fuente: Encuesta sobre Estrategias Fiscales en la Administración Municipal (CSP-UPO).

sos que hasta el momento venían desarrollando los municipios si tomamos como referencia la estructura de ingresos locales de los últimos años, esto es, la estructura institucional en la que se sitúan los municipios.

En lo que se refiere a las estrategias de reducción de gasto, los análisis han identificado cuatro tipos (Tabla 6). Por un lado, la disminución de gastos en recursos humanos, tanto en relación a su volumen (despidos o jubilaciones), como a los gastos asociados a los mismos (prestaciones, sueldos y salarios). Por otro lado, estrategias destinadas a la prestación de servicios a través de o en colaboración con otros actores, ya sean públicos (otras Administraciones), ya sean privados (empresas o entidades sin ánimo de lucro). En tercer lugar, estrategias destinadas a reducir gastos a través de recortes, bien de forma horizontal (en todas las áreas municipales), bien de forma específica (en las áreas menos eficientes o solamente en gastos administrativos que no influyen de forma tan directa en la prestación de los servicios a los ciudadanos). Finalmente, estrategias destinadas al aumento de la productividad a través de mejoras en la gestión y en los procesos de trabajo. Esta última aparecer ser la más utilizada, seguida de la de recortes de gastos, seguida por la referida a la externalización de servicios mediante la colaboración con otros actores, y por último, y minoritaria, la referida a la gestión de los recursos humanos.

Los resultados obtenidos muestran, pues, que los Ayuntamientos españoles prefieren implementar estrategias de austeridad dentro del ámbito de la Administración que otras que afecten a los empleados públicos o a la forma en que se prestan los servicios a los ciudadanos. De hecho, si se compara las medias de las estrategias de ingresos y gastos en su conjunto, puede apreciarse que se ha concedido mayor importancia a las primeras.

Este resultado es coherente con la literatura sobre estrategias de austeridad en la que se afirma que las respuestas de las Administraciones locales en tiempos de austeridad se producen de forma incremental (Levine 1981; Pammer 1990). Es decir, las Administraciones locales tratan de responder de manera paulatina, desde estrategias que produzcan menor efecto en los ciudadanos o empleados públicos, como son las de incrementos de los ingresos 


\section{TABLA 6}

Estrategias de reducción de gasto

\begin{tabular}{|c|c|c|c|}
\hline Indicadores & Variables incluidas en el indicador & $\begin{array}{c}\text { Media } \\
(0,100)\end{array}$ & $\begin{array}{c}\text { Media } \\
(0,100)\end{array}$ \\
\hline \multirow{5}{*}{ Gestión de los recursos humanos } & Despedir personal & 6,51 & \multirow{5}{*}{10,13} \\
\hline & Reducir niveles de prestaciones personales & 6,51 & \\
\hline & Congelar sueldos y salarios de empleados & 3,16 & \\
\hline & No incrementar trabajadores municipales & 23,35 & \\
\hline & Reducir personal con jubilaciones & 1,00 & \\
\hline \multirow{5}{*}{ Externalización de servicios } & Acuerdos con la Administración autonómica para prestación servicios & 25,61 & \multirow{5}{*}{15,37} \\
\hline & Acuerdos con la Administración central para la prestación de servicios & 9,13 & \\
\hline & Acuerdos con otros Ayuntamientos para la prestación de servicios & 22,07 & \\
\hline & Transferir servicios a otras Administraciones & 6,57 & \\
\hline & Prestación servicios mediante acuerdos con asociaciones & 13,50 & \\
\hline \multirow{3}{*}{ Recortes de gastos } & Recortes presupuestarios en todas las áreas & 34,29 & \multirow{3}{*}{27,86} \\
\hline & Reducir presupuesto en áreas menos eficientes & 23,63 & \\
\hline & Reducir gastos administrativos & 25,68 & \\
\hline \multirow{2}{*}{ Aumento de la productividad } & Rediseño procesos de trabajo para aumentar productividad & 40,33 & \multirow{2}{*}{38,99} \\
\hline & Mejoras en la gestión administrativa para aumentar productividad & 37,66 & \\
\hline \multicolumn{3}{|l|}{ Media total estrategias de gasto } & 23,08 \\
\hline
\end{tabular}

Fuente: Encuesta sobre Estrategias Fiscales en la Administración Municipal (CSP-UPO).

o las de incremento de productividad entre las de gasto, a estrategias más potencialmente conflictivas, como son la reducción de recursos humanos o la externalización de los servicios públicos locales. Pero además, si atendemos al desequilibrio entre competencias y recursos existente en el sistema de gobierno local español, resulta lógico pensar que las estrategias de las administraciones locales se centren en primer lugar en obtener un mayor volumen de ingresos en detrimento de las estrategias de disminución del gasto público.

\subsection{Algunas razones para la extensión de las estrategias fiscales}

Ahora bien, el sistema institucional 'afecta' más o menos por igual a todos los municipios, por lo que el mayor o menor uso de distintas estrategias debería explicarse por variaciones entre municipios, ya sea en la percepción de la situación o problemas fiscales, ya sea por las preferencias políticas de sus gobiernos, ya sea por el tamaño del municipio. Si este último informa, aunque sea indirectamente, de la capacidad del municipio para desarrollar estrategias adaptativas a su entorno, la perspectiva de la cultura política señalaría que las preferencias -o ideologíade las autoridades políticas locales podrían informar sobre el uso de unas u otras estrategias: más proclives a la no reducción de gastos mediante recortes en servicios y recursos humanos entre la izquierda, y más proclives a ello entre la derecha (Clark y Hoffman- Martinot 1998: 34). ¿Qué muestran los análisis de regresión realizados? En concreto, se han tomado como variables dependientes cada una de los tipos de estrategias fiscales delimitados an- 
teriormente, siendo variables independientes el tamaño del municipio (número de habitantes), el partido del alcalde en la escala izquierda-derecha y cada uno de lo sindicadores de problemas fiscales mostrados más arriba.

La Tabla 7 muestra los coeficientes de regresión (y significación) en cada caso, así como el coeficiente de determinación para cada análisis, esto es, para cada estrategia de ingreso y para estas en su conjunto. Puede apreciarse que, en general, las variables independientes explican poco de la variablidad en el uso de las diferentes estrategias fiscales. Solamente en el caso de las estrategias fiscales de incremento de las transferencias se llega la $11 \%$ de varianza explicada. No obstante, los resultados muestran cierta coherencia.

De forma desagregada, por tipo de estrategias, la dinámica sociopolítica del municipio, es decir, las dificultades para alcanzar acuerdos con otros municipios junto con las presiones de ciudadanos y empleados municipales, es la que en mayor medida parece explicar el desarrollo de de las estrategias fiscales de ingreso, junto con la pérdida de ingresos a la que, de forma lógica, la respuesta es el incremento en este tipo de estrategias. Se trata, pues, de la mediad en que se considera se pierden ingresos y la medida en que existen tensiones en la dinámica socio-política local, sin que el partido que encabeza el gobierno local, el tamaño del municipio u otros problemas den cuanta del uso de las estrategias de ingreso.

\section{TABLA 7}

Estrategias de aumento de ingresos: factores explicativos. Análisis de regresión linear múltiple

\begin{tabular}{|c|c|c|c|c|c|}
\hline & $\begin{array}{l}\text { Estrategias de } \\
\text { ingreso (total) }\end{array}$ & $\begin{array}{l}\text { Ingresos } \\
\text { Propios }\end{array}$ & $\begin{array}{l}\text { Incremento } \\
\text { transferencias }\end{array}$ & $\begin{array}{c}\text { Incremento } \\
\text { deuda }\end{array}$ & $\begin{array}{c}\text { Venta de bienes } \\
\text { públicos }\end{array}$ \\
\hline Variables predoctoras & \multicolumn{5}{|c|}{ Beta (significatividad) } \\
\hline Tamaño municipal & $\begin{array}{c}, 068 \\
(, 380)\end{array}$ & $\begin{array}{l}, 024 \\
(, 764)\end{array}$ & $\begin{array}{l}, 158 \\
(, 040)\end{array}$ & $\begin{array}{l}-, 063 \\
(, 426)\end{array}$ & $\begin{array}{c}, 046 \\
(, 559)\end{array}$ \\
\hline Partido político & $\begin{array}{l}-, 039 \\
(, 615)\end{array}$ & $\begin{array}{l}-, 079 \\
(, 322)\end{array}$ & $\begin{array}{l}-, 035 \\
(, 647)\end{array}$ & $\begin{array}{l}-, 038 \\
(, 628)\end{array}$ & $\begin{array}{c}, 064 \\
(, 417)\end{array}$ \\
\hline Pérdida de ingresos & $\begin{array}{l}, 146 \\
(, 096)\end{array}$ & $\begin{array}{l}, 119 \\
(, 191)\end{array}$ & $\begin{array}{c}, \mathbf{1 7 1} \\
(, 050)\end{array}$ & $\begin{array}{c}, 019 \\
(, 831)\end{array}$ & $\begin{array}{c}, 026 \\
(, 775)\end{array}$ \\
\hline Aumento de los servicios & $\begin{array}{c}, 104 \\
(, 214)\end{array}$ & $\begin{array}{l}, 090 \\
(, 296)\end{array}$ & $\begin{array}{l}-, 040 \\
(, 630)\end{array}$ & $\begin{array}{c}, 077 \\
(, 362)\end{array}$ & $\begin{array}{c}, 102 \\
(, 236)\end{array}$ \\
\hline Dinámica socioeconómica & $\begin{array}{l}-, 008 \\
(, 922)\end{array}$ & $\begin{array}{l}-, 056 \\
(, 517)\end{array}$ & $\begin{array}{c}, 097 \\
(, 245)\end{array}$ & $\begin{array}{l}, 076 \\
(, 375)\end{array}$ & $\begin{array}{l}-, 133 \\
(, 123)\end{array}$ \\
\hline Dinámica sociopolítica & $\begin{array}{l}, 149 \\
(, 081)\end{array}$ & $\begin{array}{l}-, 070 \\
(, 423)\end{array}$ & $\begin{array}{c}, 153 \\
(, 070)\end{array}$ & $\begin{array}{c}, 152 \\
(, 080)\end{array}$ & $\begin{array}{l}, 123 \\
(, 158)\end{array}$ \\
\hline $\mathrm{R} 2$ & $\begin{array}{c}, 091 \\
(, 019)\end{array}$ & $\begin{array}{l}, 028 \\
(, 620)\end{array}$ & $\begin{array}{c}, 113 \\
(, 005)\end{array}$ & $\begin{array}{c}, 060 \\
(, 024)\end{array}$ & $\begin{array}{c}, 052 \\
(, 214)\end{array}$ \\
\hline $\mathrm{N}$ & 297 & 297 & 297 & 295 & 294 \\
\hline
\end{tabular}

Fuente: Encuesta sobre Estrategias Fiscales en la Administración Municipal (CSP-UPO).

En lo que se refiere a las estrategias de gasto (Tabla 8) es, en cambio, la dinámica socioeconómica la que aparece en mayor medida junto a la dinámica sociopolítica. Ahora bien, mientras que la primera parece afectar a la extensión en la aplicación de nuevas técnicas de gestión o la externalización, como formas de reducción de gasto sin eliminar servicios, la dinámica sociopolítica lo hace respecto a estrategias 'sensibles' respecto a los empleados 


\section{TABLA 8}

Estrategias de reducción de gasto. Análisis de regresión linear múltiple

\begin{tabular}{|c|c|c|c|c|c|}
\hline & $\begin{array}{c}\text { Estrategias } \\
\text { De gasto (total) }\end{array}$ & $\begin{array}{c}\text { Gestión recursos } \\
\text { humanos }\end{array}$ & $\begin{array}{l}\text { Externalización } \\
\text { de servicios }\end{array}$ & $\begin{array}{c}\text { Reducción } \\
\text { de costes }\end{array}$ & $\begin{array}{c}\text { Aumento } \\
\text { productividad }\end{array}$ \\
\hline Variables predoctoras & \multicolumn{5}{|c|}{ Beta (significatividad) } \\
\hline Tamaño municipal & $\begin{array}{l}, 113 \\
(, 133)\end{array}$ & $\begin{array}{l}, 036 \\
(, 647)\end{array}$ & $\begin{array}{l}, 045 \\
(, 558)\end{array}$ & $\begin{array}{l}, 137 \\
(, 079)\end{array}$ & $\begin{array}{l}, 106 \\
(, 165)\end{array}$ \\
\hline Partido olítico & $\begin{array}{l}, 092 \\
(, 220)\end{array}$ & $\begin{array}{l}-, 019 \\
(, 810)\end{array}$ & $\begin{array}{l}, 052 \\
(, 493)\end{array}$ & $\begin{array}{l}, 091 \\
(, 242)\end{array}$ & $\begin{array}{l}, 113 \\
(, 140)\end{array}$ \\
\hline Pérdida de ingresos & $\begin{array}{l}, 064 \\
(, 451)\end{array}$ & $\begin{array}{l}, 023 \\
(, 795)\end{array}$ & $\begin{array}{l}, 034 \\
(, 691)\end{array}$ & $\begin{array}{l}, 137 \\
(, 121)\end{array}$ & $\begin{array}{l}-, 021 \\
(, 809)\end{array}$ \\
\hline Aumento de los servicios & $\begin{array}{l}-, 029 \\
(, 720)\end{array}$ & $\begin{array}{l}-, 055 \\
(, 524)\end{array}$ & $\begin{array}{l}-, 087 \\
(, 289)\end{array}$ & $\begin{array}{l}, 015 \\
(, 859)\end{array}$ & $\begin{array}{l}, 010 \\
(, 906)\end{array}$ \\
\hline Dinámica socioeconómica & $\begin{array}{c}, 222 \\
(, 007)\end{array}$ & $\begin{array}{c}, 064 \\
(, 463)\end{array}$ & $\begin{array}{c}, 181 \\
(, 030)\end{array}$ & $\begin{array}{c}, 069 \\
(, 416)\end{array}$ & $\begin{array}{c}, 286 \\
(, 001)\end{array}$ \\
\hline Dinámica sociopolítica & $\begin{array}{l}, 212 \\
(, 011)\end{array}$ & $\begin{array}{l}, 169 \\
(, 054)\end{array}$ & $\begin{array}{l}, 269 \\
(, 002)\end{array}$ & $\begin{array}{l}, 103 \\
(, 228)\end{array}$ & $\begin{array}{l}108 \\
, 198\end{array}$ \\
\hline R2. & $\begin{array}{l}, 138 \\
(, 001)\end{array}$ & $\begin{array}{l}, 036 \\
(, 454)\end{array}$ & $\begin{array}{l}, 116 \\
(, 004)\end{array}$ & $\begin{array}{l}, 079 \\
(, 043)\end{array}$ & $\begin{array}{l}, 114 \\
(, 004)\end{array}$ \\
\hline $\mathrm{N}$ & 297 & 297 & 297 & 297 & 295 \\
\hline
\end{tabular}

Fuente: Encuesta sobre Estrategias Fiscales en la Administración Municipal (CSP-UPO).

municipales, sea la externalización, sea, sobre todo, la gestión de los recursos humanos, en donde aparece como único factor significativo.

Ahora bien, a diferencia de otras estrategias de gasto o el conjunto de las estrategias de ingresos, el tamaño del municipio parece contar en el extensión de la estrategia orientada a reducción de costes en la producción de los servicios Por otra parte, el tamaño municipal aparece como variable predictora de las estrategias de reducción de gastos. Si, tal y como se señala más arriba le tamaño del municipio informa, aunque sea indirectamente, de la capacidad de desarrollar estrategias adaptativas al entorno, esto resultados muestran la naturaleza 'fragmentada' del sistema local español, es decir, la definición de diferentes capacidades y responsabilidades en función del tamaño municipal, y el hecho de grandes variaciones en este.

Finalmente, es destacable que en ninguno de los tipos de estrategias fiscales el partido político se muestre como un factor explicativo. A este respecto debe considerarse el modelo de gobierno local existente en España, caracterizado por un alcalde con amplias competencias tanto en lo político como en lo administrativo (Delgado et al. 1998: 240), del que podría derivarse que el partido político que gobierna en el municipio tuviera alguna capacidad explicativa en el uso de diferentes estrategias fiscales. Sin embargo, los análisis muestran resultados similares a los encontrados por Pammer (1990) para el caso de Estados Unidos en el que se señala que ello puede ser atribuido a la naturaleza de las estrategias fiscales consideradas en el análisis, las cuales están más sujetas a la decisión administrativa que al consenso del pleno municipal. En otras palabras, las medidas de austeridad consideradas parecen representar políticas internas dentro de la Administración municipal sobre las cuales los funcionarios directivos están dotados de una importante capacidad de decisión, y en donde las consideraciones derivadas de distintas 'culturas políticas' son menos importantes. 


\section{Contexto intergubernamental y dinámica socio-política municipal: sobre 'estrategias políticas' y 'estrategias administrativas'}

En general, los resultados presentados vienen a mostrar que, en correspondencia con el marco institucional que caracteriza al sistema local en España, existe una mayor aplicación de estrategias fiscales de ingreso frente a las destinadas al recorte de los gastos públicos. Y además, que entre las segundas destacan medidas de austeridad de carácter incremental, centradas en la mejora de la administración municipal, evitando aquellas que son potencialmente más conflictivas por afectar de forma directa a ciudadanos o empleados municipales. De hecho, es la dinámica socioeconómica, y sobre todo, la dinámica sociopolítica, la que en mayor medida explica la variación en el uso de las distintas estrategias.

El 'dilema de la autonomía local' orienta a los municipios hacia, por un lado, la necesidad de aumentar sus ingresos, como una estrategia política 'localista' desarrollad por todos los municipios con el objetivo de reconocimiento ('fiscal') de sus nuevas y crecientes funciones, y por otro, a tratar de reducir gastos sin desatender, sobre todo, el contexto sociopolítico en el que se encuentran, y en especial su dinámica interna, y sin que en ello cuente la cultura política del gobierno municipal. Cabría pensar pues que se estas últimas son más 'estrategias administrativas' que 'estrategias políticas', ya sea porque en ello tienen gran peso las élites administrativas y empleados municipales, ya sea porque en un contexto de aumento de responsabilidades la acción y liderazgo de las autoridades municipales cambia desde un rol de medición política a otro de gerencia de los asuntos municipales, el imperativo de la prestación de servicios (Navarro 2002). ¿Una 'nueva cultura política' entre los autoridades políticas y administrativas municipales españolas cuando se enfrentan a la gestión municipal? Parece claro que las evidencias presentadas no permiten concluirlo, siendo necesario una mayor investigación al respecto. Pero sí han mostrado la importancia del contexto institucional, que da lugar a una estrategia política localista común, y también la importancia de la dinámica sociopolítica de los municipios como factores explicativos de variaciones en la orientación de la nueva gobernanza local, considerada aquí en términos de estrategias fiscales.

Ello pudiera estar indicando que la resolución del dilema de la autonomía local no sólo es producto de los cambios en las relaciones intergubernamentales, sino también de los producidos dentro de la propia Administración local No en vano, después del aumento de ingresos (propios o de otras Administraciones), son las estrategias orientadas al aumento de la productividad en la Administración municipal las más extendida entre los municipios españoles de 10.000 o más habitantes.

\section{REFERENCIAS BIBLIOGRÁFICAS Y DOCUMENTALES}

Blanco, I. y Gomá, R. (2002), Gobiernos locales y redes participativas. Barcelona: Ariel.

Budge, I. et al. (2001), Mapping Policy Preferences. Estimates for Parties, Electors and Governments 1945-1998. Oxford: Oxford University Press.

Castle, F. y Mair, P. (1984), «Left- Right Political Scales: some «expert» judgments», European Journal of Political Research 12: 73-88.

Clark, T. N. (1994), Urban innovation. Creative strategies for turbulent times. Newbury Park, California: Sage. 
Clark, T. N. y Ferguson, L. (1983), City money: Political processes, fiscal strain and retrenchment. Nueva York: Columbia University Press.

Clark, T. N. y Hoffmant Martinot, V. (1998), The new political culture. Boulder, Colorado: Westview Press.

Consejería de Gobernación. Dirección General de Administración Local (2002), Libro Blanco de la Administración Local de Andalucia. Colección Informes y Estudios, serie Administración Local: Andalucía.

De León Ledesma, J. y Dorta Velázquez, J. (2005), «El marco de financiación de la Hacienda local en España después de la reforma", Apuntes. Revista de la Facultad de Economía (BUAP) 29.

Delgado, I. et al. (1998), "Functions and duties of Local Chief Officers», en A. Magnier y K. K. Klausen, eds., The anonymous leader. Appointed CEOs in Western Local Government. Odense: Odense University Press.

Euleau, H. y Eyestone, R. (1968), «Policy Maps of City Councils and Policy Outcomes: A developmental analysis», American Political Science Review 11: 456-78.

Glueck, W. F. (1972), Bussines Policy and Strategic Management. Nueva York: McGraw Hill.

González Caballero, J. C. y Muela Regli, N., «Municipalismo español y relaciones intergubernamentales fiscales», Documento de Trabajo. Estudios combinados de Derecho y Ciencias Politicas. Departamento de Ciencia politica y Sociología. Universidad Carlos III: Madrid.

Hambrick, D. C. (1981), «Environment, strategy and power within top management teams», Administrative Science Quarterly 2: 253-76.

Jens Blom-Hansen, L. et al. (2006), "Do parties matter for local revenue policies? A comparison of Denmark and Norway», European Journal of Political Research 45: 445-465.

Levine, C. H. (1981), The Politics of Retrenchment: How Local Government Manage Fiscal Stress. Berverly Hills: Sage Publications.

McGowan, R. D. y Stevens J. M. (1983), «Local Government Initiatives in a climate of uncertainty», Public Administration Review 41: 72-79.

Miranda, R. A (1994), «Explaining the privatization decision among governments in the United States» en T. N. Clark, ed., Urban innovation. Creative strategies for turbulent times. Newbury Park. California: Sage Publications.

Mishra, R. (1992), El Estado de Bienestar en crisis. Pensamiento y cambio social. Madrid:. Ministerio de Trabajo y Asuntos Sociales.

Mouritzen, P. E. (1992), Managing cities in austerity. Londres: Sage.

Mouritzen, P. E. y Svara, J. H. (2002), Leadership at the apex. Politicians and administrators in Western Local government. Pittsburg: University of Pittsburg Press.

Navarro Yánez, C. J. (1998), El nuevo localismo. Municipios y democracia en la sociedad actual. Córdoba: Diputación de Córdoba.

Navarro Yánez, C. J. (2002), Democracia asociativa y oportunismo politico: la politica pública de participación ciudadana en los municipios españoles (1979-1993). Valencia. Tirant lo Blanch. 
Navarro Yánez, C. J. (2006), Ciudadanía y Administración Municipal. Descentralización y centros civicos. La visión de la ciudadanía cordobesa. Córdoba: Ayuntamiento de Córdoba.

Navarro Yánez, C. J. y Ramírez Pérez, A (2003), «The structure of opportunities to new localism and 'glocalization': mesolevel political and cultural factors", en F. Entrena, ed., Local reaction to Globalization Processes. Nueva York: Nova Science Publishers Inc.

Nivola, P. (1982), «Apocalipse now? Whither the Urban Fiscal Crisis», Polity 14: 371-94.

Offe, C. (1990), Contradicciones del Estado de Bienestar. Madrid: Alianza.

Page, E. y Goldsmith, M. (1987), Central and Local government relation. Beverly Hills: Sage.

Pammer, W. J. (1990), Managing Fiscal Strain in Major American Cities. Understanding Retrenchment in the public sector. Nueva York: Greenwood Press.

Stein, R. M. (1984), «State regulations and the political consequences of urban fiscal stress», The Journal of Federalism 14. Filadelfia: CSF Associates.

Suárez Pandiello, J. (2001), «El futuro de la financiación local en el contexto del Estado de las Autonomías», Hacienda Pública Española: 359-378.

Ylönen, D. (1989), «Local leadership and bureucratic legitimicy: the Finish case», en S. Clarke, ed., Urban Innovation and Autonomy. Londres: Sage. 


\section{ANEXOS}

Para validar cada uno de los indicadores sintéticos utilizados en el texto se han realizado dos análisis. Por un lado, factorial de componentes principales, que da cuenta de la dimensionalidad de las respuestas de los entrevistados. Varianza explicadas iguales o superiores al $60 \%$ permiten validar tal dimensionalidad. Por otro lado, para cada factor resultante se han analizado las correlaciones inter-items para determinar la validez de su elaboración como sumatorio. Se ofrecen aquí los resultados de tales análisis. En concreto, la Tabla A para problemas fiscales, la Tabla $\mathrm{B}$ para estrategias de ingreso y la Tabla $\mathrm{C}$ para estrategias de gasto.

\section{TABLA A}

Percepción problemas fiscales: análisis factorial (matriz de componentes rotada) y correlaciones inter-items

\begin{tabular}{|c|c|c|c|c|c|c|}
\hline & \multicolumn{4}{|c|}{ Componente } & \multirow{2}{*}{$\begin{array}{l}\text { Correlaciones } \\
\text { inter-ítem }\end{array}$} \\
\hline & & 1 & 2 & 3 & 4 & \\
\hline \multirow{4}{*}{ Pérdida de ingresos } & Pérdida de ingresos del gobierno central &, 872 & & & & \multirow{4}{*}{,383 } \\
\hline & Pérdida de ingresos del gobierno autonómico &, 757 & & & & \\
\hline & Disminución de ingresos propios &, 470 & & & & \\
\hline & Limitación de ingresos o gastos &, 554 & & & & \\
\hline \multirow{2}{*}{ Aumento de los servicios } & Aumento demanda servicios por los ciudadanos & &, 816 & & & \multirow{2}{*}{,264 } \\
\hline & Costes por la transferencia de servicios al municipio & &, 579 & & & \\
\hline \multirow{2}{*}{ Dinámica socioeconómica } & La inflación & & &, 793 & & \multirow{2}{*}{,314 } \\
\hline & El desempleo & & &, 863 & & \\
\hline \multirow{3}{*}{ Dinámica sociopolítica } & Imposibilidad de acuerdos con otros municipios & & & &, 812 & \multirow{3}{*}{,377 } \\
\hline & Presiones de los contribuyentes & & & &, 726 & \\
\hline & Presiones de empleados municipales & & & &, 594 & \\
\hline \multicolumn{2}{|c|}{ Varianza explicada acumulada } & 18,35 & 34,03 & 48,10 & 61,97 & \\
\hline
\end{tabular}

Método de extracción: Análisis de componentes principales.

Rotación: Normalización Varimax con Kaiser.

Solamente se incluyen factores con saturación igual o superior a 30.

Fuente: Encuesta de Estrategias Fiscales en la Administración Municipal. CSP-UPO. 


\section{TABLA B}

Estrategias de aumento de ingress: análisis factorial (matriz de componentes rotada) y correlaciones inter-items

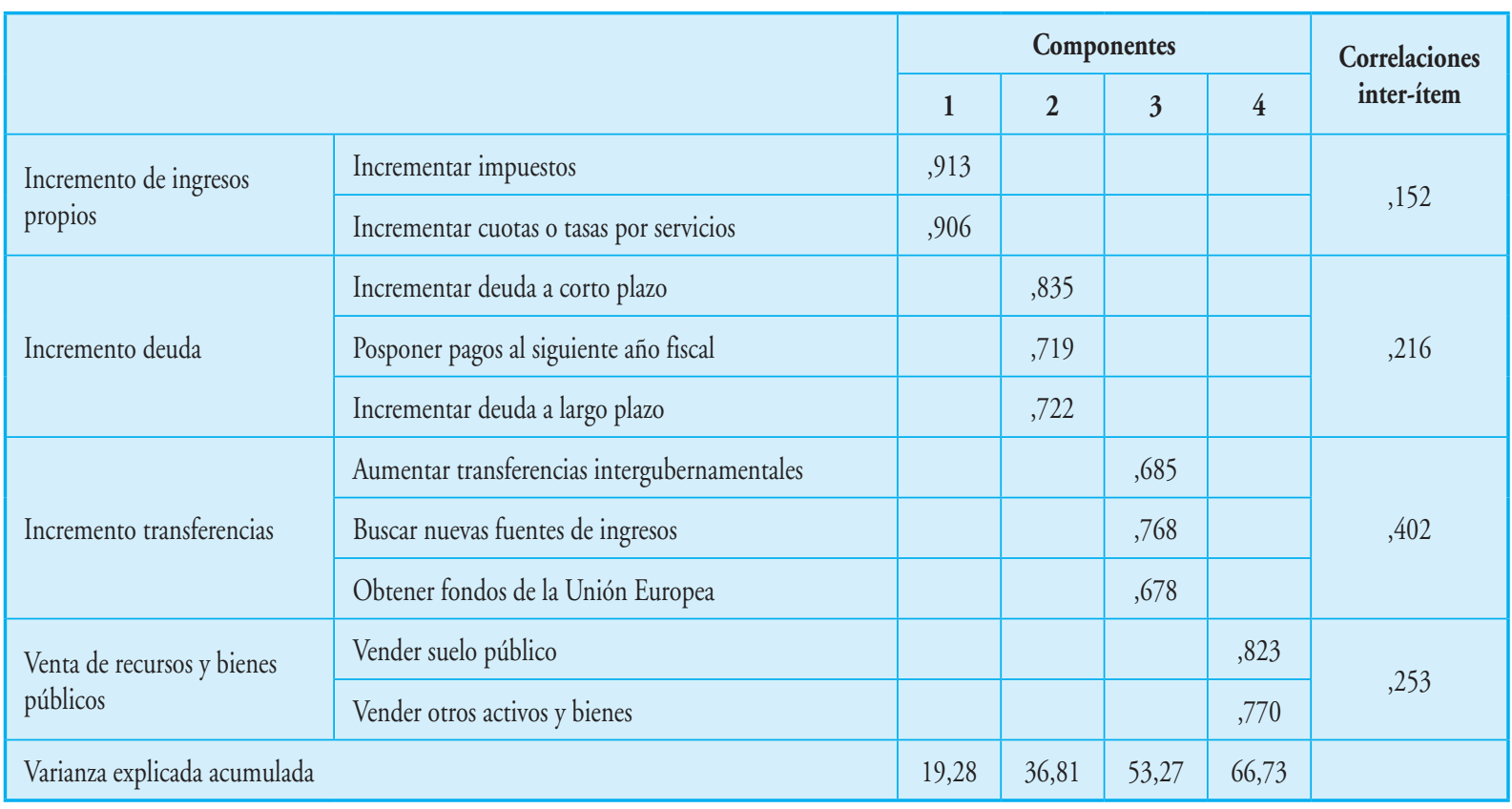

Método de extracción: Análisis de componentes principales. Rotación: Normalización Varimax con Kaiser. Solamente se incluyen factores con saturación igual o superior a 30.

Fuente: Encuesta de Estrategias Fiscales en la Administración Municipal. CSP-UPO. 


\section{TABLA C}

Estrategias de reducción de gastos: análisis factorial (matriz de componentes rotada) y correlación inter-items

\begin{tabular}{|c|c|c|c|c|c|c|}
\hline & \multicolumn{4}{|c|}{ Componente } & \multirow{2}{*}{$\begin{array}{l}\text { Correlaciones } \\
\text { inter-ítem }\end{array}$} \\
\hline & & 1 & 2 & 3 & 4 & \\
\hline \multirow{5}{*}{$\begin{array}{l}\text { Gestión de los } \\
\text { recursos humanos }\end{array}$} & Despedir personal & ,707 & &, 427 & & \multirow{5}{*}{,425 } \\
\hline & Reducir niveles de prestaciones personales & ,753 & & & & \\
\hline & Congelar sueldos y salarios de empleados &, 747 & & & & \\
\hline & No incrementar trabajadores municipales & ,636 & & & & \\
\hline & Reducir personal con jubilaciones &, 548 & & & & \\
\hline \multirow{6}{*}{$\begin{array}{l}\text { Externalización de } \\
\text { servicios }\end{array}$} & Acuerdos con admón. autonómica para prestación servicios & &, 772 & & & \multirow{6}{*}{, 465} \\
\hline & Acuerdos con admón. central para la prestación de servicios &, 322 & ,683 & & & \\
\hline & Acuerdos con otros ayuntamientos para prestar servicios & &, 629 & & & \\
\hline & Transferir servicios a otras administraciones &, 325 &, 528 & & & \\
\hline & Prestación servicios con asociaciones & & ,680 & & & \\
\hline & Prestación servicios con empresas privadas & & ,628 & & & \\
\hline \multirow{3}{*}{ Recortes de gastos } & Recortes presupuestarios en todas las áreas & & & 840 & & \multirow{3}{*}{,440 } \\
\hline & Reducir presupuesto en áreas menos eficientes & & & ,648 & & \\
\hline & Reducir gastos administrativos & 369 & &, 595 &, 324 & \\
\hline \multirow{2}{*}{$\begin{array}{l}\text { Aumento de la } \\
\text { productividad }\end{array}$} & Rediseño procesos de trabajo para aumentar productividad & & & & ,918 & \multirow{2}{*}{,395 } \\
\hline & Mejoras en gestión administrativa para aumentar productividad & & & & ,898 & \\
\hline \multicolumn{2}{|c|}{ Varianza explicada acumulada } & 20,23 & 36,43 & 50,76 & 62,14 & \\
\hline
\end{tabular}

Método de extracción: Análisis de componentes principales.

Rotación: Normalización Varimax con Kaiser.

Solamente se incluyen factores con saturación igual o superior a 30.

Fuente: Encuesta de Estrategias Fiscales en la Administración Municipal. CSP-UPO. 